\title{
Simple surface singularities
}

\author{
Jan Stevens
}

\begin{abstract}
By the famous ADE classification, rational double points are simple. Rational triple points are also simple. We conjecture that the simple normal surface singularities are exactly those rational singularities whose resolution graph can be obtained from the graph of a rational double point or rational triple point by making any number of vertex weights more negative. We show that no other rational singularities can be simple. We prove simpleness only for special classes of singularities, namely rational quadruple points or sandwiched singularities.
\end{abstract}

\section{Introduction}

Simple hypersurface singularities were classified by Arnol'd in the famous ADE list [Arn72]. In the surface case these are exactly the rational double points. In Giusti's list [Giu83] of simple isolated complete intersection singularities, no surface singularities occur. They do appear in the classification of simple determinantal codimension two singularities by Frühbis-Krüger and Neumer [FKN10]; the simple surface singularities are the rational triple points.

In this paper we address the following question.

QUESTION 1.1. What are the simple normal surface singularities?

The singularities that are considered are germs of complex spaces, and here simple means that only finitely many isomorphism classes occur in the versal deformation. The known cases suggest that simple singularities are rational, with star-shaped graph. But not all rational singularities are simple: already for rational quadruple points, a cross ratio can be involved in the exceptional divisor.

Conjecture 1.2. Simple normal surface singularities are exactly those rational singularities whose resolution graphs can be obtained from the graphs of rational double points and rational triple points by making any number of vertex weights more negative.

Such a graph determines the analytic type of the singularity; this property is called tautness. The graphs of taut singularities were classified by Laufer [Lau73]. The star-shaped ones make up the parts I, II and III in his list, and, as Laufer observed, they are precisely the ones that can be obtained from double-point and triple-point graphs.

A rigid singularity, one having no nontrivial deformations at all, is certainly simple. It is an old unsolved question whether rigid normal surface singularities exist. Our conjecture says that

Received 29 November 2013, accepted in final form 10 May 2016.

2010 Mathematics Subject Classification 32S25 (primary), 14J17, 32S30 (secondary).

Keywords: simple singularities, rational singularities, tautness, sandwiched singularities.

This journal is (C) Foundation Compositio Mathematica 2017. This article is distributed with Open Access under the terms of the Creative Commons Attribution Non-Commercial License, which permits non-commercial reuse, distribution, and reproduction in any medium, provided that the original work is properly cited. For commercial re-use, please contact the Foundation Compositio Mathematica. 


\section{Simple SURFACE SINGULARITIES}

they do not. Without the condition of normality, there are rigid singularities. In fact, the standard example of a nonnormal isolated singularity, two planes in 4-space meeting transversally in one point, is rigid and therefore simple.

Our results on the conjecture are restricted to rational singularities. We prove one direction of the conjecture for rational singularities in general, that those not covered by the conjecture are not simple. We show that they deform to a singularity with a cross ratio on the exceptional set, by studying deformations of the resolution. Proving simpleness is more difficult. We succeed in the cases where there are good methods to study deformations. We prove the following.

Proposition 1.3. For the following classes of rational singularities, the conjecture is true; that is, the simple rational singularities are those obtained from rational double and triple points:

- quotient singularities

- rational quadruple points

- sandwiched singularities

The case of quotient singularities is already known; it follows by combining the results of [Bri68] and [EV85]. For rational quadruple points, the first case after double and triple points, we have quite a good understanding of the versal deformation by the work of De Jong and Van Straten [dJvS91]. They also studied the deformation theory of sandwiched singularities [dJvS98], which are normal surface singularities admitting a birational map to $\left(\mathbb{C}^{2}, 0\right)$.

Not all quotient singularities are sandwiched. If the graph of a singularity contains $D_{4}$ or the graph of Proposition 5.5 as subgraph, then it is not sandwiched. This applies in particular to singularities of Laufer's type III.5 (see Table 1) and higher.

As singularities of Laufer's type III.5 (see Table 1) are deformations of those of type III.6, and those of types III.7 and III.8 are deformations of singularities of type III.9, it remains to prove that singularities of type III.9 or III. 6 and those of type III. 4 which are not sandwiched are simple.

\section{The conjecture}

By a singularity $X$ we always mean the germ $(X, p)$ of a complex analytic space around a point $p \in X$. If $X$ is an isolated singularity, then there exists a versal deformation $\left(X_{S}, p\right) \rightarrow(S, 0)$ with special fibre $X_{0}$ isomorphic to $X$. It makes sense to talk about the isomorphism class of a nearby fibre $X_{s}$ with $0 \neq s \in S$.

DEFinition 2.1. A singularity is simple if only finitely many different isomorphism classes of singularities occur in its versal deformation.

The simple 2-dimensional hypersurface singularities were classified by Arnol'd in the famous ADE list. They are also known under many other names, each representing a different aspect, leading to different generalisations. As Kleinian singularities they are quotients of $\mathbb{C}^{2}$ by finite subgroups of $\mathrm{SL}(2, \mathbb{C})$. Non-hypersurface singularities are obtained by taking quotients by subgroups of $\mathrm{GL}(2, \mathbb{C})$; these quotient singularities were classified by Brieskorn [Bri68]. They are examples of rational surface singularities.

We recall some notions which will be important later on. General references are [Bri68, Ném99, Rei97]. Let $\pi:(\widetilde{X}, E) \rightarrow(X, p)$ be a resolution of a normal surface singularity with as exceptional set $E$ a normal crossings divisor. Then $R^{1} \pi_{*} \mathcal{O}_{\widetilde{X}}$ is a finite-dimensional vector space, 


\section{J. Stevens}

whose dimension is the geometric genus $p_{g}$ of $X$. A singularity $X$ is rational if $p_{g}(X)=0$. This condition can be checked from the resolution graph. By a result of Artin, the singularity is rational if and only if $p_{a}(D) \leqslant 0$ for any positive cycle $D$ on $E$, where the arithmetic genus of a cycle can be computed with the adjunction formula. It suffices that $p_{a}(Z)=0$ for the fundamental cycle, which is by definition the unique minimal positive cycle $Z$ such that $Z \cdot E_{i} \leqslant 0$ for every irreducible component $E_{i}$ of $E$.

The fundamental cycle can be found with a computation sequence [Lau72]: let $Z_{1}$ be any component of $E$, and define inductively $Z_{j+1}=Z_{j}+E_{i(j)}$, where $E_{i(j)} \cdot Z_{j}>0$, until there is no component of $E$ intersecting the obtained cycle positively and $Z$ has been reached. In a computation sequence the genus cannot go down:

$$
p_{a}\left(Z_{j+1}\right)=p_{a}\left(Z_{j}\right)+p_{a}\left(E_{i(j)}\right)+Z_{j} \cdot E_{i(j)}-1 \geqslant p_{a}\left(Z_{j}\right) .
$$

This gives an algorithmic rationality criterion.

Lemma 2.2 (Laufer's criterion). A singularity is rational if $p_{a}\left(Z_{j+1}\right)=0$ in every step $Z_{j+1}$ of a computation sequence, so $E_{i(j)} \cdot Z_{j}=1$ in every step.

For rational singularities, the resolution graph contains much information about the analytic structure of the singularity. In particular, the multiplicity is equal to $-Z \cdot Z$, and the embedding dimension is $1-Z \cdot Z$. Therefore the only rational hypersurface singularities are the rational double points.

Rational triple points are also simple. This follows from the classification of simple determinantal codimension 2 singularities by Frühbis-Krüger and Neumer [FKN10]. Nonrational singularities of this type are not simple.

But not all rational singularities are simple. Already for quadruple points, we find singularities with a modulus in the resolution. An example is the $n$-star singularity of [dJvS91], which has a star-shaped graph with a central (-4)-vertex and four arms of $(-2)$-vertices of equal length $n-1$. On the other hand, there exist simple singularities of arbitrary multiplicity, as quotient singularities are simple. This has been stated explicitly in the unpublished Diploma thesis of Matthias Kabel (Hamburg, 2004). It follows from two facts: quotient singularities deform only into other quotient singularities [EV85], and the analytic type of a quotient singularity is determined by the resolution graph [Bri68].

Definition 2.3. A normal surface singularity is taut if every other singularity with the same resolution graph is isomorphic to it. A singularity is pseudotaut if there are only finitely many isomorphism classes of singularities with the same resolution graph.

Laufer classified all taut and pseudotaut surface singularities [Lau73]. The only nonrational ones are the minimally elliptic singularities whose graph is a Kodaira graph and those are not simple.

Actually, Laufer defines a singularity as pseudotaut if there are countably many isomorphism classes with the same graph, but he proves that there are then only finitely many, as a result of a more precise description. For a pseudotaut graph, all analytic types occur in a certain versal deformation: using the standard plumbing construction, construct a manifold $M$ with exceptional divisor $E$, and consider deformations of the pair $(M, E)$ which are locally trivial deformations of $E$. For the general fibre, all such deformations are in fact trivial. For all other singularities, the resolution has moduli. This does not imply that the singularity itself has moduli: deformations of the resolution blow down to deformations of the singularity if and only if the geometric genus $p_{g}$ 
is constant. As the geometric genus is semi-continuous, this last condition is always satisfied for rational singularities. In particular, pseudotautness is a necessary condition for the simpleness of a rational singularity. We show below (Proposition 3.6) that pseudotaut but not taut rational singularities are not simple.

We conjecture that simple singularities are rational. The evidence is not only the fact that all known simple singularities are indeed rational but also the behaviour of the next much-studied class of singularities after the rational ones. Minimally elliptic singularities deform into the cone over an elliptic curve of the same multiplicity (where the multiplicity of the cone is the degree of the curve) [Kar83] and are therefore never simple. We expect this behaviour to be typical.

There is one further restriction on the graphs of the known simple normal surface singularities: the graph is always star-shaped. The graph of a surface singularity is star-shaped whenever the singularity is quasi-homogeneous, that is, admits a good $\mathbb{C}^{*}$-action. Moreover, given a star-shaped graph, there always exists a quasi-homogeneous singularity with this graph [OW71]. So a taut singularity with star-shaped graph is quasi-homogeneous.

This leads us to the following conjecture.

Conjecture 2.4. A normal surface singularity is simple if and only if it is taut and quasihomogeneous.

Remark 2.5. Quasi-homogeneity is a feature of many lists of simple objects, but in each case it is the result of the classification. In fact, the list [BG82] of simple map germs $(\mathbb{C}, 0) \rightarrow\left(\mathbb{C}^{2}, 0\right)$ also contains germs which are not quasi-homogeneous.

In Table 1, we list all star-shaped graphs from Laufer's list of taut singularities [Lau73]. It is organised as to have no duplicates. The meaning of the symbols is the following: A dot - denotes a vertex of any weight $-b \leqslant-2$, a dot ${ }^{-2}$ has weight exactly -2 , whereas a square $\mathbf{\square}$ is a vertex of any weight $-b \leqslant-3$, that is, less than -2 . A chain $-\cdots \longrightarrow$ is a chain of vertices of any length $k \geqslant 0$. So the first entry of the table gives exactly the cyclic quotient singularities, with the cone over a rational normal curve being the case $k=0$. The second entry gives all other quasi-homogeneous taut singularities with reduced fundamental cycle.

From the table one sees that the graphs can be characterised as obtainable from the graphs of rational double points and triple points, as Laufer already observed. This gives the equivalent form of our conjecture, which was stated in the introduction.

Conjecture 2.6. Simple normal surface singularities are exactly those rational singularities whose resolution graphs can be obtained from the graphs of rational double points and rational triple points by making any number of vertex weights more negative.

We will say for short that the singularities are obtainable from rational double and triple points.

\section{Deformations on the Artin component}

The deformation space of a rational surface singularity has a special component, of largest dimension, over which a simultaneous resolution exists (after base change). Therefore deformations on this component can be found from deformations of the resolution. These were studied by Laufer and Wahl [Lau79, Wah79]. Their theory leads to sufficient conditions for the existence of a deformation in terms of the combinatorics of cycles on the resolution. 


\section{J. Stevens}

TABLE 1. Quasi-homogeneous taut surface singularities

I, II

III.1

III. 2

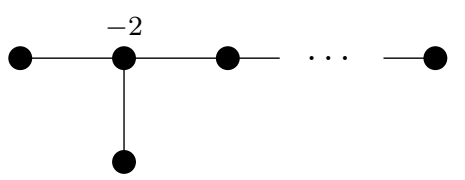

III.3

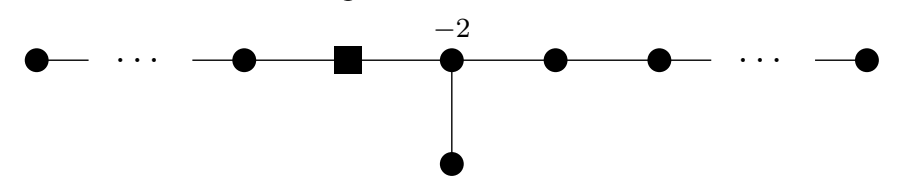

III.4

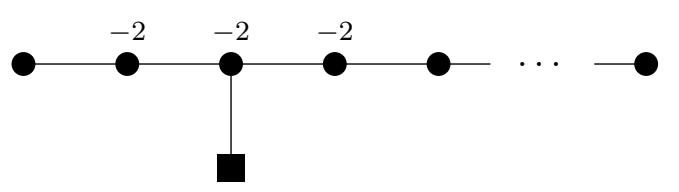

III.5

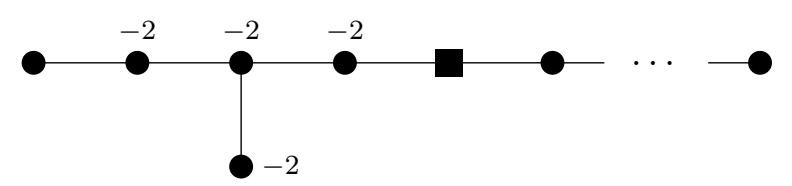

III.6

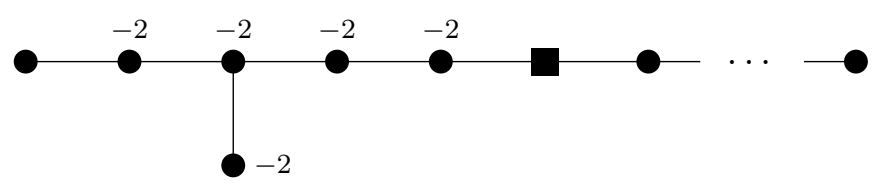

III.7

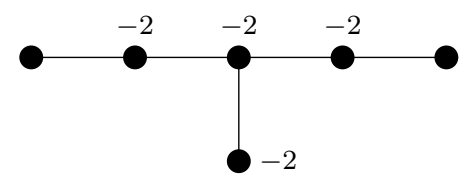

III. 8

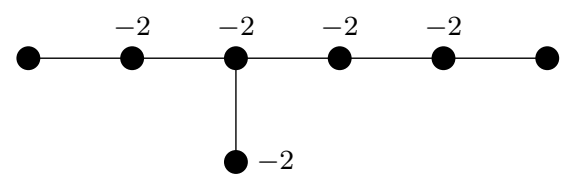

III.9

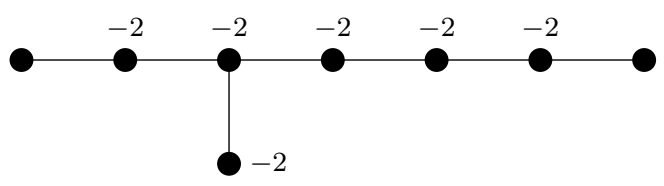




\section{Simple SURFACE SINGULARITIES}

Let $X_{T} \rightarrow T$ be a 1-parameter deformation of $X_{0} \cong X$ with simultaneous resolution $\widetilde{X}_{T} \rightarrow T$. Consider an irreducible component $E_{T} \rightarrow T$ of the exceptional set mapping surjectively onto $T$. Then $E_{T}$ is flat and proper over $T$. The fibre $E_{t}$ for $t \neq 0$ is irreducible [Lau79, Theorem 2.1]; in fact, if there are several components, there is monodromy and we find two components which are homologous in $\widetilde{X}_{T}$, contradicting the negative definiteness of the intersection form on $X_{t}$. In the family, $E_{t}^{2}$ and $p_{a}\left(E_{t}\right)$ are constant. For $t=0$, the divisor $E_{t}$ specialises to a cycle $D$ on the exceptional set of $X_{0}$. We say that $D$ lifts to the deformation $\widetilde{X}_{T} \rightarrow T$. Laufer and Wahl give sufficient conditions on cycles $D$ for the existence of a deformation to which $D$ lifts. To formulate the result, we need some definitions.

Definition 3.1 ([Wah79]). A cycle $D>0$ on the minimal resolution of a rational singularity is a positive root if $p_{a}(D)=0$.

For the ADE singularities, the positive roots correspond exactly to the positive roots of the root system of the same type A, D or E under the identification of the resolution graph with the Dynkin diagram. For general rational singularities, Laufer's criterion (Lemma 2.2) leads to an algorithmic characterisation of the roots.

Lemma 3.2 ([Lau79, Lemma 3.1]). A cycle $D>0$ is a positive root if and only if $D$ is part of a computation sequence.

Definition 3.3. A cycle $D=\sum d_{i} E_{i}$ on the exceptional set of the minimal resolution of a normal surface singularity is almost reduced if it is reduced at the non- $(-2)$-vertices, that is, $d_{i}=1$ if $E_{i}^{2}<-2$.

This condition is important, as it implies the vanishing of the obstructions to deform $D$, which lie in $H^{1}\left(\mathcal{T}_{D}^{1}\right)$, because $h^{1}\left(\mathcal{T}_{D}^{1}\right)=h^{1}\left(\mathcal{O}_{D-D_{\text {red }}}(D)\right)=\left(D-D_{\text {red }}\right) \cdot K$ [Wah79, (2.14)].

Definition 3.4 ([Lau79, Definition 3.5]). A collection of cycles $D_{1}, \ldots, D_{m}$ on the exceptional set of the minimal resolution of a rational surface singularity is integrally minimal if each $D_{i}$ is a positive root, the cycle $D=D_{1}+\cdots+D_{m}$ is almost reduced, and no other collection $C_{1}, \ldots, C_{m}$ of $m$ positive roots can generate the $D_{i}$ with nonnegative integral coefficients.

Theorem 3.5. Let $D_{1}, \ldots, D_{m}$ be an integrally minimal collection of cycles on the minimal resolution $\widetilde{X}_{0}$ of a rational surface singularity with $D=\sum D_{i}$ itself a positive root. Then there exists a 1-parameter deformation $\widetilde{X}_{T} \rightarrow T$ such that the exceptional set $E_{t}$ of $\widetilde{X}_{t}$ for $t \neq 0$ has a decomposition in $m$ irreducible components $E_{t, i}$ with $E_{t, i}$ homologous to $D_{i}$ in $\widetilde{X}_{T}$.

If the fundamental cycle $Z$ of $\widetilde{X}_{0}$ is itself almost reduced, then every deformation arises this way.

Proof. The result is contained in [Lau79, Theorem 3.12]. Note that Laufer has almost reduced fundamental cycle as assumption, but his proof shows that the existence of an integrally minimal collection is sufficient for the existence of a deformation.

Some deformations, which Laufer calls reduced, are particularly simple. In terms of the resolution graph, they are the following. First of all, one can take a (connected) subgraph of a given graph. One can also replace two intersecting curves $E_{a}$ and $E_{b}$ of self-intersection $-a$ and $-b$, respectively, with one curve with the same self-intersection as $E_{a}+E_{b}$, that is $-(a+b-2)$. This corresponds to smoothing the double point of the exceptional divisor at the intersection point of $E_{a}$ and $E_{b}$. 


\section{J. Stevens}

TABLE 2. Confining nonsimple singularities

$$
\widetilde{E}_{6}
$$

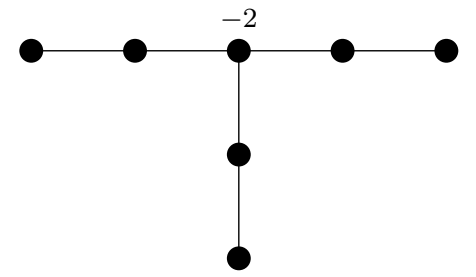

$\widetilde{E}_{7}$

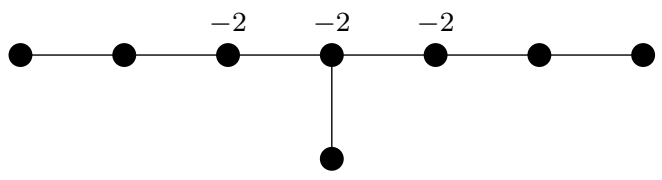

$\widetilde{E}_{8}$

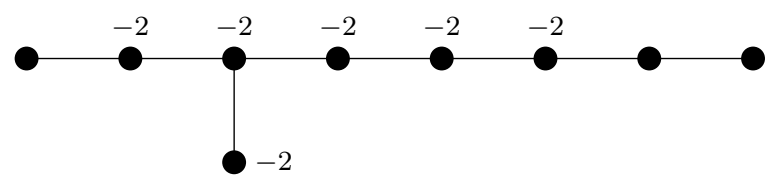

Proposition 3.6. A rational singularity which is not obtainable from a rational double or triple point is not simple. It deforms into a singularity with a modulus in the exceptional divisor, with (unweighted) graph of the form

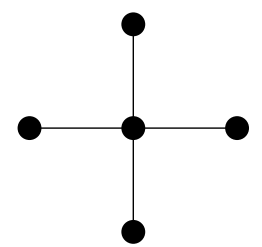

Proof. For the purpose of this proof we call a graph as in the statement a star. If the graph of a rational singularity has a vertex of valency at least 4 , then it has a star as subgraph. If there are two vertices $E_{a}$ and $E_{b}$ of valency 3 , then we can smooth all double points of the exceptional divisor on the chain between $E_{a}$ and $E_{b}$. In terms of the graph, we combine all vertices on the chain, including $E_{a}$ and $E_{b}$, into one new vertex. The new graph has a star as subgraph.

We are left with graphs with exactly one vertex of valency 3 . We claim that every such graph which is not obtained from a double or triple point graph has as subgraph a rational graph of form given in Table 2. The meaning of the symbols is the same as in Table 1. If all vertex weights are -2 , then the graph is $\widetilde{E}_{6}, \widetilde{E}_{7}$ or $\widetilde{E}_{8}$ (also known as the Kodaira graphs IV*, III* or II*). One has to make appropriate vertex weights more negative to obtain rational graphs. We refer to the resulting graphs as graphs of type $\widetilde{E}_{k}$. To prove the claim, one has only to carefully inspect Table 1 and note which graphs are absent. First of all, the central curve has to be a (-2)-curve. If the three arms all have length at least 3 (counting from the central vertex), there is a subgraph of type $\widetilde{E}_{6}$. Otherwise, if there is one arm of length 2 and the other two have length at least 4 , then the two vertices on the long arms next to the central vertex have to be $(-2)$-vertices, and there is a subgraph of type $\widetilde{E}_{7}$. If not, the second arm has to have length 3 , and the third arm has length at least 6 , and there are at least six $(-2)$-curves, so there is a subgraph of type $\widetilde{E}_{8}$. The proposition follows from the following lemma.

Lemma 3.7. Rational singularities with a graph of type $\widetilde{E}_{k}$ deform into a star. 


\section{Simple SURFACE SINGULARITIES}

Proof. We need some notation. We denote the central vertex by $E_{0}$. There are three arms, of length $p, q$ and $r$ (counted from the central vertex). Here $(p, q, r)=(3,3,3),(2,4,4)$ or $(2,3,6)$. In terms of Table 2, the number $p$ is the length of the arm pointing downwards and $r$ the length of the right arm. The arms are $E_{1,1}+\cdots+E_{1, p-1}, E_{2,1}+\cdots+E_{2, q-1}$ and $E_{3,1}+\cdots+E_{3, r-1}$, with $E_{i, j}^{2}=-b_{i, j}$ and $E_{i, 1}$ intersecting $E_{0}$.

In each of the three cases we define a collection $\left\{D_{0}, \ldots, D_{4}\right\}$ of integrally minimal cycles, such that the graph of the collection is a star with $D_{0}$ as central vertex, that is, $D_{0} \cdot D_{i}=1$ and $D_{i} \cdot D_{j}=0$ for $1 \leqslant i<j \leqslant 4$. Theorem 3.5 then gives the existence of a deformation into a star with the given graph. For graphs of type $\widetilde{E}_{6}$ we take

$$
\begin{array}{ll}
D_{0}=E_{0}+E_{1,1}+E_{2,1}+E_{3,1}, & D_{0}^{2}=-\left(b_{1,1}+b_{2,1}+b_{3,1}-4\right), \\
D_{i}=E_{i, 2}, \quad 1 \leqslant i \leqslant 3, & D_{i}^{2}=-b_{i, 2}, \\
D_{4}=E_{0}, & D_{4}^{2}=-2 ;
\end{array}
$$

for $\widetilde{E}_{7}$ we take

$$
\begin{array}{ll}
D_{0}=E_{1,1}+E_{2,2}+E_{2,1}+E_{0}+E_{3,1}+E_{3,2}, & D_{0}^{2}=-\left(b_{1,1}+b_{2,2}+b_{3,2}-4\right), \\
D_{1}=E_{0}+E_{2,1}, & D_{1}^{2}=-2, \\
D_{2}=E_{0}+E_{3,1}, & D_{2}^{2}=-2, \\
D_{3}=E_{2,3}, & D_{3}^{2}=-b_{2,3}, \\
D_{4}=E_{3,3}, & D_{4}^{2}=-b_{3,3} ;
\end{array}
$$

and for $\widetilde{E}_{8}$ we take

$$
\begin{array}{ll}
D_{0}=E_{2,2}+E_{2,1}+E_{0}+E_{3,1}+E_{3,2}+E_{3,3}+E_{3,4}, & D_{0}^{2}=-\left(b_{2,2}+b_{3,4}-2\right), \\
D_{1}=E_{1,1}+E_{0}+E_{2,1}, & D_{1}^{2}=-2, \\
D_{2}=E_{1,1}+E_{0}+E_{3,1}+E_{3,2}+E_{3,3}, & D_{2}^{2}=-2, \\
D_{3}=E_{1,1}+E_{2,1}+2 E_{0}+2 E_{3,1}+E_{3,2}, & D_{3}^{2}=-2, \\
D_{4}=E_{3,5}, & D_{4}^{2}=-b_{3,5} .
\end{array}
$$

Corollary 3.8. Any nonsimple rational singularity deforms into a star.

Proof. If a singularity obtainable from a rational double or triple point only deforms into such singularities (necessarily only finitely many), then it is simple. So if it is not simple, then it deforms to a rational singularity that is not of this type, and therefore also into a star.

Next, we study deformations on the Artin component of quasi-homogeneous taut singularities. If the singularity is obtainable from a double point and the multiplicity is at least 4, then it can also be obtained from a triple point. Note that it can deform into double points. We start by determining the positive roots.

Lemma 3.9. Let $X^{\prime}$ be obtained from the rational triple point $X$, with the irreducible components $E_{i}^{\prime}$ of the exceptional divisor of $X^{\prime}$ corresponding to the components $E_{i}$ of $X$. A cycle $D^{\prime}=\sum d_{i} E_{i}^{\prime}$ is a positive root of $X^{\prime}$ if and only if $D=\sum d_{i} E_{i}$ is a positive root of $X$ with $d_{i}=1$ for all $i$ with $\left(E_{i}^{\prime}\right)^{2}<E_{i}^{2}$.

Proof. We first show that an $E_{i}^{\prime}$ with $\left(E_{i}^{\prime}\right)^{2}=E_{i}^{2}-\beta_{i}<E_{i}^{2}$ has coefficient 1 in the fundamental cycle. We construct $X^{\prime}$ from $X$ by blowing up $\beta_{i}$ smooth points of $E$. The exceptional divisor $E^{\prime}$, 


\section{J. Stevens}

which is the strict transform of $E$, is a subset of the exceptional set (the total transform of $E$ ) of a nonminimal resolution of $X$. The fundamental cycle on this resolution can be computed by first computing the fundamental cycle on $E^{\prime}$. By Laufer's criterion (Lemma 2.2), each (-1)-curve intersects this cycle with multiplicity 1 . Therefore the condition on $d_{i}$ is necessary.

Let $D^{\prime}$ be a positive root, so $p_{a}\left(D^{\prime}\right)=0$. We compute $p_{a}(D)=1+\frac{1}{2} D \cdot(D+K)$. If $d_{i}>1$, then $E_{i} \cdot D=d_{i} E_{i}^{2}+\sum_{E_{i} \cdot E_{j}>0} d_{j}=d_{i}\left(E_{i}^{\prime}\right)^{2}+\sum_{E_{i}^{\prime} \cdot E_{j}^{\prime}>0} d_{j}=E_{i}^{\prime} \cdot D^{\prime}$ and $E_{i} \cdot K=E_{i}^{\prime} \cdot K^{\prime}$. If $d_{i}=1$ and $\left(E_{i}^{\prime}\right)^{2}=E_{i}^{2}-\beta_{i}$, then $E_{i} \cdot(D+K)=E_{i}^{\prime} \cdot D^{\prime}+\beta_{i}+E_{i}^{\prime} \cdot K^{\prime}-\beta_{i}=E_{i}^{\prime} \cdot\left(D^{\prime}+K^{\prime}\right)$. So also $p_{a}(D)=0$. The same computation shows that $p_{a}(D)=0$ implies $p_{a}\left(D^{\prime}\right)=0$ if $d_{i}=1$ whenever $\beta_{i}>0$.

Proposition 3.10. A singularity obtainable from a rational triple point deforms on the Artin component only into singularities obtainable from triple and double points.

Proof. Let $X^{\prime}$ deform into a surface with several singularities. By the openness of versality, we can smooth all but one of them, so we may as well assume that there is only one singularity. As $X^{\prime}$ has an almost reduced fundamental cycle, the deformation $X_{T}^{\prime}$ can be described by an integrally minimal collection $D_{1}^{\prime}, \ldots, D_{m}^{\prime}$ of positive roots. Lemma 3.9 gives an integrally minimal collection $D_{1}, \ldots, D_{m}$, determining a deformation $X_{T}$ of the triple point $X$. As triple points deform only into triple or double points, the graph of $D$ is a double- or triple-point graph. An $E_{i}^{\prime}$ with $\left(E_{i}^{\prime}\right)^{2}=E_{i}^{2}-\beta_{i}<E_{i}^{2}$ can occur in at most one $D_{j}^{\prime}$, as its coefficient in $D^{\prime}$ is 1 . Therefore, $\left(D_{j}^{\prime}\right)^{2}=D_{j}^{2}-\sum \beta_{i}(j)$, where the sum runs over all $i$ such that $E_{i}^{\prime}$ is contained in the support of $D_{j}$. So the graph of $D^{\prime}$ is obtainable from that of $D$.

\section{Rational quadruple points}

The deformation theory of rational quadruple points was studied by De Jong and Van Straten [dJvS91]. Up to a smooth factor, the base space of the versal deformation is isomorphic to an explicitly described space $B(n)$ with $n+1$ irreducible components. The integer $n$ can be found from the resolution graph of the rational quadruple points, as it is the number of virtual quadruple points or, in other words, the number of quadruple points in the resolution process.

If the base space has only two components, then a deformation to any other quadruple point has to occur over the intersection of the two components. So, in particular, it is a deformation on the Artin component.

Proposition 4.1. A rational quadruple point obtained from a triple point is simple.

Proof. By Proposition 3.10 any other quadruple point on the Artin component is obtainable from a triple point. Therefore simpleness follows if the base space is $B(1)$, with exactly two components.

We show that $n=1$, that is, there is no quadruple point on the first blow-up. Let $X^{\prime}$ be obtained from the triple point $X$, and let $E_{0}$ be the (-3)-curve of $X$ and $E_{m}^{\prime}$ the unique curve of the quadruple point $X^{\prime}$ with $E_{m}^{\prime} \cdot E_{m}^{\prime}<E_{m} \cdot E_{m}$ (possibly $E_{m}=E_{0}$ ). There is a quadruple point on the first blow-up of $X^{\prime}$ if and only if $E_{i}^{\prime} \cdot Z^{\prime}=0$ for every curve $E_{i}^{\prime}$ on the chain from $E_{0}^{\prime}$ to $E_{m}^{\prime}$.

The multiplicity of $E_{0}^{\prime}$ in the fundamental cycle $Z^{\prime}$ is 1 . If $E_{0}^{\prime} \cdot Z^{\prime}=0$ on $\tilde{X}^{\prime}$, then the neighbour $E_{1}^{\prime}$ of $E_{0}^{\prime}$ on the chain has the same multiplicity in $Z^{\prime}$ as $E_{1}$ in $Z$, and if $E_{1}^{\prime} \cdot Z^{\prime}=0$, its neighbour has also the same multiplicity, and so on. This process either stops with an $E_{i}^{\prime}$ such that $E_{i}^{\prime} \cdot Z^{\prime}<0$ or reaches $E_{m}^{\prime}$, where $E_{m}^{\prime}$ has the same multiplicity in $Z^{\prime}$ as $E_{m}$ does in $Z$. But then $E_{m}^{\prime} \cdot Z^{\prime}<0$. 


\section{Simple SURFACE SINGULARITIES}

Remark 4.2. We can characterise the simple rational quadruple points as those with almost reduced fundamental cycle, so that the singularities on the first blow-up of the singularity have multiplicity at most 3 . The easiest way to see this is to check the classification of quadruple points [Ste91, Proposition 4], as it is not always immediately obvious which of the two ( -3$)$-curves one has to make into a $(-2)$ to get a triple point.

\section{Sandwiched singularities}

Sandwiched singularities are normal surface singularities which admit a birational map to $\left(\mathbb{C}^{2}, 0\right)$. We study them and their deformations following De Jong and Van Straten [dJvS98].

A sandwiched singularity can be constructed in the following way. Start with a curve germ in the plane and resolve it in such a way that the total transform of the curve is a normal crossings divisor. The blow-up now contains the resolution of a surface singularity: its exceptional divisor is a maximal connected subset of the exceptional locus not containing (-1)-curves. This construction is not unique, but the main point is that it relates surface singularities to plane curves.

For the theory of plane curve singularities, we refer to [BK81, Chapter 8], which gives a detailed description of the equivalence of the following data: the topological type, the resolution graph (of the embedded resolution), the system of multiplicity sequences and finally the Puiseux pairs of the branches and their intersection multiplicities.

To better describe sandwiched singularities, De Jong and Van Straten [dJvS98] equip curve singularities with extra information. For each branch this is a number, bounded below in a simple way by the multiplicities of the infinitely near singularities appearing in the resolution process.

Definition 5.1. Let $C=\bigcup_{i \in B} C_{i}$ be a plane curve singularity. The number $m(i)$ is the sum of the multiplicities of the branch $C_{i}$ in the multiplicity sequence of the minimal embedded resolution of $C$.

Definition 5.2. A decorated curve is a curve singularity together with a function $l: B \rightarrow \mathbb{N}$ on the set of branches with the property that $l(i) \geqslant m(i)$. A decorated curve $(C, l)$ is nonsingular if $C$ consists of one smooth branch and $l(1)=0$.

Definition 5.3. Let $(C, l)$ be a singular decorated curve on a smooth surface $(Z, p)$, and let $m_{i}$ be the multiplicity of the $i$ th branch $C_{i}$. Consider the blow-up $\mathrm{Bl}_{p} Z \rightarrow Z$ of the singular point. The strict transform $(\bar{C}, \bar{l})$ of $(C, l)$ is the decorated curve consisting of the strict transform $\bar{C}$ of $C$ with decoration $\bar{l}(i)=l(i)-m_{i}$.

Note that we do not allow blow-ups in nonsingular decorated curves, for then $\bar{l}$ would become a negative function. Therefore the embedded resolution of a decorated curve is the unique (minimal) composition of point blow-ups such that the strict transform of the decorated curve is nonsingular. It is obtained from the minimal embedded resolution of $C$ by $l(i)-m(i)$ consecutive point blow-ups in each branch $C_{i}$.

DeFinition 5.4. Let $(C, l) \subset(Z, p)$ be a decorated curve with embedded resolution $(\bar{C}, 0) \subset$ $\widetilde{Z}(C, l)$. The analytic space $X(C, l)$ is obtained from $\widetilde{Z}(C, l)-\bar{C}$ by blowing down the maximal compact subset, that is, all exceptional divisors not intersecting the strict transform $\bar{C}$.

The space $X(C, l)$ can be smooth, or it may have several singularities. Each singularity is a sandwiched singularity. Given a sandwiched singularity, it is always possible to find a decorated 


\section{J. Stevens}

curve $(C, l)$ such that the sandwiched singularity is the only singularity of the space $X(C, l)$. Even then the representation of a sandwiched singularity as $X(C, l)$ is not unique.

Proposition 5.5. A singularity is not sandwiched if its graph contains $D_{4}$ as subgraph or has the following subgraph:

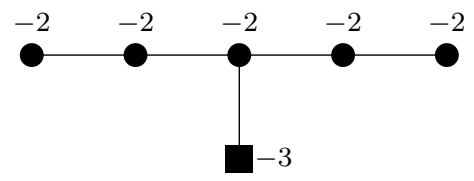

Proof. A sandwiched singularity has at least one exceptional curve $E_{i}$ with $E_{i} \cdot Z<0$ and multiplicity 1 in the fundamental cycle $Z$. Indeed, the strict transform of the first blown-up curve in the construction has this property: the compact part of the divisor of a general linear function is an upper bound for $Z$. This criterion excludes $D_{4}$ and the shown graph.

It follows that the singularities of type III.5 and higher in Table 1 are not sandwiched. Most of the other ones are not excluded by the above criterion, and in fact they are sandwiched.

We now consider deformations. The numbers $l(i)$ determine a divisor on the normalisation $\bar{C}$ of $C$. This interpretation allows a more global point of view, in which a decorated curve $(C, l)$ is a curve $C$ on a smooth surface $Z$ together with a divisor $l$ on the normalisation $\bar{C}$.

Definition 5.6. A 1-parameter deformation $\left(C_{S}, l_{S}\right)$ of a decorated curve $(C, l)$ over a germ $S$ of a smooth curve is a deformation $C_{S} \rightarrow S$ of $C$ with simultaneous normalisation $\bar{C}_{S} \rightarrow S$, together with a deformation of the divisor $l$, such that each fibre $\left(C_{s}, l_{s}\right)$ is a decorated curve.

Theorem 5.7 ([dJvS98, Theorem 4.4]). The 1-parameter deformations of a sandwiched singularity $X(C, l)$ are exactly deformations $X\left(C_{S}, l_{S}\right)$ for 1-parameter deformations $\left(C_{S}, l_{S}\right)$ of the decorated curve $(C, l)$.

A simplified proof can be found in the $\mathrm{PhD}$ thesis of Möhring [Möh04], who constructs the deformation $X\left(C_{S}, l_{S}\right)$ directly from $\left(C_{S}, l_{S}\right)$ by blowing up a family of complete ideals.

The power of the theorem comes from the fact that one has quite a good understanding of deformations with simultaneous normalisation. By a result of Teissier, these are exactly the deformations where the $\delta$-invariant (the number of virtual double points) is constant (for a proof, see [GLS07, II.2.6]). In particular, the intersection number of two branches is constant in such a deformation.

To study deformations of taut sandwiched singularities, we use a specific representation with a decorated curve. We start with the classes I, II and III.1, which consist of singularities with reduced fundamental cycle. For these classes, see also [dJvS98, Example 1.5(4)]. We construct the resolution graph of a decorated curve. This is an embedded resolution graph for the curve $C$, with as usual arrows for the strict transforms $\bar{C}_{i}$ and decorations $\bar{l}(i)=0$, which we omit. We choose one end of the resolution graph of the singularity, whose vertex $E_{0}$ will correspond to the strict transform of the first curve blown up. To a vertex $E_{i}$ with $i \neq 0$, we connect $-Z \cdot E_{i}$ copies of a $(-1)$-vertex, each with an arrow attached to it. To $E_{0}$, we connect one less vertex and arrow, namely only $-Z \cdot E_{i}-1$.

EXAMPLE 5.8 (The cyclic quotient singularity $X_{37,11}$ ). We start at the left end. The resulting 


\section{Simple SURFACE SINGULARITIES}

graph is

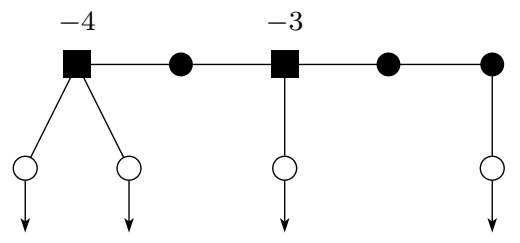

It blows down to the following decorated curve:

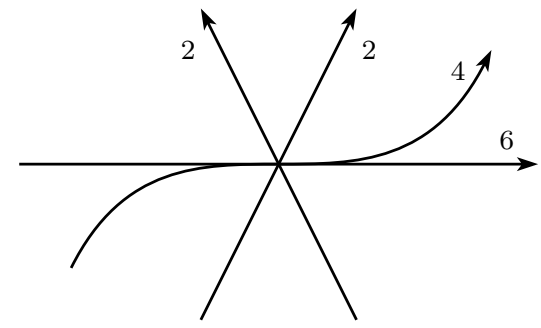

For cyclic quotient singularities, this representation with smooth branches has the property that $\min \{l(i), l(j)\}=C_{i} \cdot C_{j}+1$ for each pair of branches. Möhring [Möh04] observed that this property can be used to give a new proof of Riemenschneider's conjecture that cyclic quotients deform only into cyclic quotients. Kollár and Shepherd-Barron [KSB88] derive the conjecture from the stronger result that in a deformation of a rational singularity with reduced fundamental cycle, the number of ends of the graph cannot go up. Their result can also be obtained with the present methods. We first treat the cyclic quotient case, where the argument is more transparent.

Lemma 5.9. Let $(C, l)$ be the germ of a decorated curve with smooth branches with the property that for each pair of branches

$$
C_{i} \cdot C_{j} \leqslant \min \{l(i), l(j)\} \leqslant C_{i} \cdot C_{j}+1 .
$$

Then the only singularities of the space $X(C, l)$ are cyclic quotients.

Proof. Let $C_{r}$ be a branch such that $l(i) \leqslant l(r)$ for all branches $C_{i}$. We construct the embedded resolution of $(C, l)$ in two steps. We first consecutively blow up $l(r)$ times in the origin of the strict transform of the branch $C_{r}$. This introduces a chain $E_{1}, \ldots, E_{l(r)}$ of exceptional curves. If $l(i)=C_{i} \cdot C_{r}+1$, we blow up once in the intersection point of $E_{l(i)}$ and the strict transform of $C_{i}$, and we do not blow up further in $C_{i}$. The newly introduced (-1)-curve intersects the strict transform of $C_{i}$ on the minimal resolution and is therefore not part of the exceptional set for $X(C, l)$. If $l(i)=C_{i} \cdot C_{r}$, we do not blow up in $C_{i}$, and the curve $E_{l(i)}$ does not belong to the exceptional set. This set is thus a subset of the chain $E_{1}, \ldots, E_{l(r)-1}$, which may consist of several connected components.

Proposition 5.10. Cyclic quotient singularities deform only into cyclic quotients.

Proof. Choose, as above, a representation $X(C, l)$ with $(C, l)$ a decorated curve with smooth branches and the property that $\min \{l(i), l(j)\}=C_{i} \cdot C_{j}+1$ for each pair of branches. Let $X(\widetilde{C}, \tilde{l})$ be a general fibre of a 1-parameter deformation. Consider a pair of branches. Suppose $C_{i} \cdot C_{j}=n$ and $l(i)=n+1$. Then $\widetilde{C}_{i} \cdot \widetilde{C}_{j}=\sum_{p} n_{p}=n$, where the sum runs over the intersection points. The support of $\tilde{l}(i)$ on $\widetilde{C}_{i}$ may contain other points. Now

$$
\sum_{p \in \widetilde{C}_{i} \cap \widetilde{C}_{j}} \tilde{l}_{p}(i)+\sum_{q \notin \widetilde{C}_{j}} l_{q}(i)=l=n+1=1+\sum_{p \in \widetilde{C}_{i} \cap \widetilde{C}_{j}} n_{p} .
$$




\section{J. Stevens}

Because $\tilde{l}_{p}(i) \geqslant n_{p}$ for all $p$, we see that $\tilde{l}_{p}(i)=n_{p}+1$ holds for at most one point while for the other points, $\tilde{l}_{p}(i)=n_{p}$. So for each singularity $p$ of $(\widetilde{C}, \tilde{l})$, the property $\left(\widetilde{C}_{i} \cdot \widetilde{C}_{j}\right)_{p} \leqslant$ $\min \left\{\tilde{l}_{p}(i), \tilde{l}_{p}(j)\right\} \leqslant\left(\widetilde{C}_{i} \cdot \widetilde{C}_{j}\right)_{p}+1$ holds.

Lemma 5.11. Let $(C, l)$ be the germ of a decorated curve with smooth branches. Suppose that the set of branches $B$ can be written as the (not necessarily disjoint) union $B_{1} \cup \cdots \cup B_{k}$ such that for all $1 \leqslant m \leqslant k$, the property

$$
C_{i} \cdot C_{j} \leqslant \min \{l(i), l(j)\} \leqslant C_{i} \cdot C_{j}+1
$$

holds for all pairs $(i, j) \in B_{m} \times B_{m}$. Then the number of ends of the singularities of the space $X(C, l)$ is at most $k+1$.

Proof. Again we construct the embedded resolution of $(C, l)$ in two steps. For each subset $B_{m}$, we choose a branch $C_{m}$ with $l(m)$ maximal. The first step is to resolve the curve $\cup_{m} C_{m}$. As this curve has $k$ branches, the resulting embedded resolution graph has (at most) $k+1$ ends. The exceptional curves of the additional blow-ups needed to resolve $(C, l)$ are not exceptional for $X(C, l)$.

Proposition 5.12. In a deformation of a rational singularity with reduced fundamental cycle, the number of ends cannot increase.

Proof. We choose a representation with a decorated curve with smooth branches. For each end of the graph of the singularity (except the root), we choose a curve $C_{m}$ whose strict transform is connected to this end by a $(-1)$-curve. The set $B_{m}$ contains all branches which are connected by $(-1)$-curves to the chain from the root to $C_{m}$. Then $\min \{l(i), l(j)\}=C_{i} \cdot C_{j}+1$ for all $i, j \in B_{m}$. As before, we deduce that $\left(\widetilde{C}_{i} \cdot \widetilde{C}_{j}\right)_{p} \leqslant \min \left\{\tilde{l}_{p}(i), \tilde{l}_{p}(j)\right\} \leqslant\left(\widetilde{C}_{i} \cdot \widetilde{C}_{j}\right)_{p}+1$ for each singular point of the deformed curve $(\widetilde{C}, \tilde{l})$ through which branches in the set $B_{m}$ pass. Therefore we have a partition in at most $k$ sets and each singularity of $X(\widetilde{C}, \tilde{l})$ has at most $k+1$ ends.

COROLlary 5.13. Singularities with graph of type III.1 deform only to singularities of type I, II or III.1.

Remark 5.14. For a nonreduced fundamental cycle, the number of ends can increase. The deformations of Lemma 3.7 provide examples. We give a sandwich description of the deformation for the case of a surface singularity of type $\widetilde{E}_{6}$ with $-b_{1,1}=-4$ and all other self-intersections equal to -2 . It has a sandwiched representation with decorated curve $\left(E_{12}, 12\right)$, where $E_{12}$ is the curve $x^{3}+y^{7}+a x y^{5}=0$. It deforms into $\left(\widetilde{E}_{7},(4,4,4)\right)$, giving a 2 -star.

Next, we study sandwiched singularities in the classes III.2 and III.3. The singularities of type III.2 whose graph contains a $D_{4}$ subgraph are not sandwiched, but they are simple as they are dihedral quotients. The sandwiched ones can be seen as special case of the type III.3 if we allow the arms to be shorter. This means that we are looking at graphs of the form

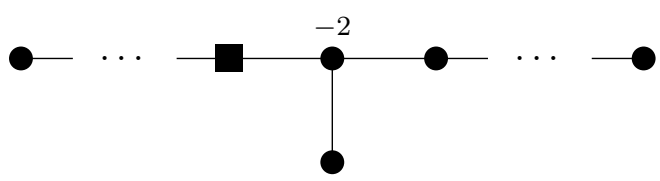

Proposition 5.15. Sandwiched singularities with graph as above deform only to singularities of the same type or to singularities with reduced fundamental cycle and at most three ends. 


\section{Simple SURFACE SINGULARITIES}

Proof. We start by describing a decorated curve $(C, l)$. We need some notation. The left arm of the graph is $E_{1,1}+\cdots+E_{1, k}$, with $E_{1, k}$ the $(-3)$-vertex. The right arm is $E_{2,1}+\cdots+E_{2, s+1}$ and the short arm consists only of $E_{3,1}$. First, look at the triple point graph of this form, which has exactly one $(-3)$-vertex and further only $(-2)$-vertices. A decorated curve giving this graph is $\left(A_{2 k}, 2 k+4+s\right)$. We call the curve $C_{0}$. To make the self-intersections more negative, we add branches. They come in three types, one for each arm. On the left arm, if a (-1)-vertex intersects $E_{1, m}$, then we have a smooth branch $C_{i}$ with $C_{0} \cdot C_{i}=2 m$ and $l\left(C_{i}\right)=m+1$. We make the short arm $E_{3,1}$ more negative with a smooth branch $C_{i}$ with $C_{0} \cdot C_{i}=2 k+1$ and $l\left(C_{i}\right)=k+2$. Finally, if a $(-1)$ intersects $E_{2, n}$ on the right arm, then we have a branch $C_{i}$ of type $A_{2 k}$ with $C_{0} \cdot C_{i}=4 k+2+n$ and $l\left(C_{i}\right)=2 k+3+n$.

We use induction on the number of branches of $(C, l)$. If the curve consists only of $\left(C_{0}, l(0)\right)$, the claim is true, as an $A_{2 k}$ has a $\delta$-const deformation into one $A_{2 l}$ with $0 \leqslant l<k$ and some $A_{2 m_{i}-1}$ with $k=l+\sum m_{i}$. The curve $\left(A_{2 l}, 2 l+1+t\right)$ gives at most a smaller graph of the same type, while $X\left(A_{2 m-1},\left(m+t_{1}, m+t_{2}\right)\right)$ has reduced fundamental cycle and at most three ends.

Now, consider a deformation $X(\widetilde{C}, \tilde{l})$ of $X(C, l)$ where $C$ has several branches. Let $C_{i}$ be a branch different from $C_{0}$. We wish to compare the singularities of $X(\widetilde{C}, \tilde{l})$ and $X(\widetilde{C} \backslash \widetilde{C}, \tilde{l})$. The proposition follows from the following claim.

Claim 5.16. The graph of the singularities of $X(\widetilde{C}, \tilde{l})$ is a subgraph of the graph of $X\left(\widetilde{C} \backslash \widetilde{C}_{i}, \tilde{l}\right)$ or is obtainable from a subgraph by making some self-intersections more negative.

We first resolve the decorated curve $\left(\widetilde{C} \backslash \widetilde{C}_{i}, \tilde{l}\right)$. If this resolution also resolves $(\widetilde{C}, \tilde{l})$, that is, no further blow-ups on the strict transform of $\widetilde{C}_{i}$ are needed, then the exceptional curves intersected by the strict transform of $\widetilde{C}_{i}$ are not exceptional for $X(\widetilde{C}, \tilde{l})$, and the graph of the singularities of $X(\widetilde{C}, \tilde{l})$ is a subgraph of the graph of $X\left(\widetilde{C} \backslash \widetilde{C}_{i}, \tilde{l}\right)$.

We shall show that if this is not the case, then exactly one extra blow-up is needed. If the centre of the blow-up does not lie on an exceptional divisor, we get just one (-1)-curve and no new singularity. If the centre is a smooth point on an irreducible component $E_{a}$ of the exceptional divisor, then the self-intersection $E_{a} \cdot E_{a}$ is made more negative. The graph of the singularity in question is therefore obtainable from a subgraph of the graph of $X\left(\widetilde{C} \backslash \widetilde{C}_{i}, \tilde{l}\right)$. Finally, if the centre is an intersection point of two divisors $E_{a}$ and $E_{b}$, then as the newly introduced (-1)-curve is not exceptional for $X(\widetilde{C}, \tilde{l})$, it breaks up the graph; the self-intersections of $E_{a}$ and $E_{b}$ become more negative. Therefore, the graphs for $X(\widetilde{C}, \tilde{l})$ are obtainable from subgraphs of the graph of $X\left(\widetilde{C} \backslash \widetilde{C}_{i}, \tilde{l}\right)$.

We look only at the intersection of a branch $\widetilde{C}_{i}$ with $\widetilde{C}_{0}$. If $C_{i}$ is a smooth branch with $C_{0} \cdot C_{i}=2 m$ and $l\left(C_{i}\right)=m+1$, then one has to blow up in $m+1$ points to resolve $\left(\widetilde{C}_{i}, m+1\right)$. Let $m_{0, p}$ be the multiplicity of $\widetilde{C}_{0}$ in such a point $p$. As $\widetilde{C}_{0} \cdot \widetilde{C}_{i}=\sum_{p} m_{0, p}=2 m$, it follows that either $m_{0, p}=2$ for $m$ points and one point does not lie on $\widetilde{C}_{0}$, or all $m+1$ points lie on $\widetilde{C}_{0}$ and for two of them $m_{0, p}=1$. A similar argument settles the case of a smooth branch $C_{i}$ with $C_{0} \cdot C_{i}=2 k+1$ and $l\left(C_{i}\right)=k+2$.

Finally, we look at a branch $C_{i}$ of type $A_{2 k}$ with $C_{0} \cdot C_{i}=4 k+2+n$ and $l\left(C_{i}\right)=2 k+3+n$. To resolve $\left(\widetilde{C}_{i}, l\left(\widetilde{C}_{i}\right)\right)$ we need $k+3+n$ blow-ups, and $\widetilde{C}_{i}$ has multiplicity 2 in $k$ of them. In these points the multiplicity of $\widetilde{C}_{0}$ can be 2,1 or 0 . As the intersection multiplicity $\widetilde{C}_{0} \cdot \widetilde{C}_{i}$ is $4 k+2+n$, there are only two possibilities. The first one is $k \times 4+(n+1) \times 1+0$, with the same numbers as for $C_{0} \cdot C_{i}$. The second possibility is that in one multiplicity 2 point of $\widetilde{C}_{i}$, the multiplicity of $\widetilde{C}_{0}$ is 1 ; then $4 k+2+n=(k-1) \times 4+2+2+(n+2) \times 1$. 


\section{J. Stevens}

Therefore in all cases at most one blown-up point does not lie on the strict transform of $\widetilde{C}_{0}$.

Singularities with a graph of type III.4 are not sandwiched if the graph has the graph of Proposition 5.5 as subgraph. For such singularities to be sandwiched, some vertex weights on this subgraph have to be more negative. We distinguish three types, with different sandwich representation. Let the left arm be $E_{1,2}+E_{1,1}$, where we always have $-b_{1,1}=-2$, let the short arm be $E_{3,1}$, and let the right arm be $E_{2,1}+E_{2,2}+\cdots+E_{2, k}$. For each of the three types, the singularity of lowest multiplicity is a quadruple point, realisable as $X\left(C_{0}, l\right)$ with $C_{0}$ irreducible.

For the first type, $-b_{3,1}=-4$ (for the quadruple point). We take $\left(E_{6}, k+7\right)$ as decorated curve. To make the self-intersection of $E_{3,1}$ (the first blown-up curve) more negative, we add one or more smooth branches with $l=2$, for $E_{1,2}$ we add smooth branches with $l=3$, and finally for $E_{2, t}$ on the right arm, we add one or more branches $\left(E_{6}, t+7\right)$ intersecting $C_{0}$ with multiplicity $12+t$.

The second type always has $-b_{3,1}=-3$. If we also have $-b_{1,2}=-3$, we get the quadruple point from the irreducible curve $\left(E_{8}, k+8\right)$. To make $E_{1,2} \cdot E_{1,2}$ more negative, we add one or more smooth branches with $l=2$, and for $E_{2, t}$ on the right arm, we add one or more branches $\left(E_{8}, t+8\right)$ intersecting $C_{0}$ with multiplicity $15+t$.

The last type always has $-b_{3,1}=-3$ and $-b_{1,2}=-2$. In this case, $E_{2, k}$ is the first blown-up curve. For the quadruple point, the only other (-3)-curve besides $E_{3,1}$ is $E_{2,2}$. We take a curve which is equisingular to $\left(x^{3}+y^{3 k-1}, 4+3 k\right)$; here we may assume $k>2$, as $k=2$ was dealt with in the previous case. The only curves which can be made more negative are on the right arm, and we use smooth branches for this purpose.

Proposition 5.17. Sandwiched singularities with graph of type III.4 deform only to singularities of type I, II or III.1 up to III.4.

Proof. We again use induction on the number of branches. For the induction start, we have to describe $\delta$-const deformations of $\left(C_{0}, l\right)$. For $E_{6}$ and $E_{8}$ this is not difficult, but for the third type it is not so easy. Therefore we use a different argument. By Proposition 4.1, quadruple points obtainable from triple points are simple. As sandwiched singularities deform only into sandwiched singularities, we get the statement of the proposition.

For the induction step, we use the same claim as in the proof of Proposition 5.15. As before, the result follows if we can prove that either the resolution $\left(\widetilde{C} \backslash \widetilde{C}_{i}, \tilde{l}\right)$ is also the resolution of $(\widetilde{C}, \tilde{l})$ or only one extra blow-up in a smooth point of the strict transform of $\widetilde{C}_{i}$ is needed. For smooth branches this is the same argument as before. It remains to look at branches of types $E_{6}$ and $E_{8}$. As these cases are similar, we only discuss the last one.

If $E_{8}$ deforms $\delta$-const to a collection of $A_{k}$-singularities, then the multiplicities of the (infinitely near) points in the minimal embedded resolution are $(2,2,2,2)$, whereas they are $(3,2)$ if there is a triple point. This can be checked from the list of possible combinations; it is all the information we need here. Therefore, the multiplicities in the resolution of $\left(E_{8}, t+8\right)$ are $\left(3,2,1^{t+3}\right)$ or $\left(2^{4}, 1^{t}\right)$. The intersection multiplicity with $\widetilde{C}_{0}$ has to be $15+t$. If the multiplicities in the minimal resolution of $\widetilde{C}_{0}$ are $(3,2)$, then we can get $15+t$ as $9+4+(t+2)+0$ or $9+2+2+(t+2)$, but not from a $\widetilde{C}_{i}$ with multiplicities $\left(2^{4}, 1^{t}\right)$, as $6+4+2+2+t=14+t$. If $\widetilde{C}_{0}$ is of type $\left(2^{4}\right)$, then we can get $6+4+2+2+(t+1)$, or $4 \times 4+(t-1)+0$ or $3 \times 4+2+2+(t-1)$. So there is at most one smooth point of the strict transform of $\widetilde{C}_{i}$ on the resolution of $\left(\widetilde{C}_{0}, \tilde{l}\right)$, which still has to be blown up. 


\section{Simple SURFACE SINGULARITIES}

Corollary 5.18. Sandwiched singularities are simple if and only if they are taut and quasihomogeneous.

\section{REFERENCES}

Arn72 V.I. Arnol'd, Normal forms of functions near degenerate critical points, the Weyl groups $A_{k}$, $D_{k}, E_{k}$ and Lagrangian singularities, Funkcional. Anal. i Priložen. 6 (1972), no. 4, 3-25, http: //mi.mathnet.ru/faa2531 (Russian), Funct. Anal. Appl. 6 (1972), no. 4, 254-272 (English); https://doi.org/10.1007/BF01077644.

BG82 J.W. Bruce and T. J. Gaffney, Simple singularities of mappings $\mathbb{C}, 0 \rightarrow \mathbb{C}^{2}, 0$, J. London Math. Soc. 26 (1982), no. 3, 465-474; https://doi.org/10.1112/jlms/s2-26.3.465.

BK81 E. Brieskorn and H. Knörrer, Ebene algebraische Kurven (Birkhäuser Verlag, Basel - Boston, Mass., 1981); https://doi.org/10.1007/978-3-322-80311-5.

Bri68 E. Brieskorn, Rationale Singularitäten komplexer Flächen, Invent. Math. 4 (1968), 336-358; https://doi.org/10.1007/BF01425318.

EV85 H. Esnault and E. Viehweg, Two-dimensional quotient singularities deform to quotient singularities, Math. Ann. 271 (1985), no. 3, 439-449; https://doi.org/10.1007/BF01456078.

FKN10 A. Frühbis-Krüger and A. Neumer, Simple Cohen-Macaulay codimension 2 singularities, Comm. Algebra 38 (2010), no. 2, 454-495; https://doi.org/10.1080/00927870802606018.

Giu83 M. Giusti, Classification des singularités isolées simples d'intersections complètes, Singularities, Part 1 (Arcata, Calif., 1981), Proc. Sympos. Pure Math., vol. 40 (Amer. Math. Soc., Providence, RI, 1983), 457-494; https://doi.org/10.1090/pspum/040.1/713086.

GLS07 G.-M. Greuel, C. Lossen, and E. Shustin, Introduction to singularities and deformations, Springer Monogr. Math. (Springer, Berlin, 2007); https://doi.org/10.1007/3-540-28419-2.

dJvS91 T. de Jong and D. van Straten, On the base space of a semi-universal deformation of rational quadruple points, Ann. of Math. 134 (1991), no. 3, 653-678; https://doi.org/10.2307/ 2944359.

dJvS98 _ Deformation theory of sandwiched singularities, Duke Math. J. 95 (1998), no. 3, 451-522; https://doi.org/10.1215/S0012-7094-98-09513-8.

Kar83 U. Karras, Normally flat deformations of rational and minimally elliptic singularities, Singularities, Part 1 (Arcata, Calif., 1981), Proc. Sympos. Pure Math., vol. 40 (Amer. Math. Soc., Providence, RI, 1983), 619-639; https://doi.org/10.1090/pspum/040.1/713098.

KSB88 J. Kollár and N. I. Shepherd-Barron, Threefolds and deformations of surface singularities, Invent. Math. 91 (1988), no. 2, 299-338; https://doi.org/10.1007/BF01389370.

Lau72 H. B. Laufer, On rational singularities, Amer. J. Math. 94 (1972), 597-608; https://doi.org/ $10.2307 / 2374025$.

Lau73 , Taut two-dimensional singularities, Math. Ann. 205 (1973), 131-164; https://doi. org/10.1007/BF01350842.

Lau79 Ambient deformations for exceptional sets in two-manifolds, Invent. Math. 55 (1979), no. 1, 1-36; https://doi.org/10.1007/BF02139700.

Möh04 K. Möhring, On sandwiched singularities, Ph.D. thesis, Mainz, 2004, available at http:// archimed.uni-mainz.de/pub/2004/0015/diss.pdf.

Ném99 A. Némethi, Five lectures on normal surface singularities, Low Dimensional Topology (Eger, 1996/Budapest, 1998), Bolyai Soc. Math. Stud., vol. 8 (János Bolyai Math. Soc., Budapest, 1999), 269-351.

OW71 P. Orlik and P. Wagreich, Isolated singularities of algebraic surfaces with $\mathbf{C}^{*}$ action, Ann. of Math. 93 (1971), 205-228; https://doi.org/10.2307/1970772. 


\section{J. Stevens}

Rei97 M. Reid, Chapters on algebraic surfaces, Complex Algebraic Geometry (Park City, UT, 1993), IAS/Park City Math. Ser., vol. 3 (Amer. Math. Soc., Providence, RI, 1997), 3-159.

Ste91 J. Stevens, Partial resolutions of rational quadruple points, Internat. J. Math. 2 (1991), no. 2, 205-221; https://doi.org/10.1142/S0129167X91000144.

Wah79 J. M. Wahl, Simultaneous resolution and discriminantal loci, Duke Math. J. 46 (1979), no. 2, 341-375; https://doi.org/10.1215/S0012-7094-79-04615-5.

Jan Stevens stevens@chalmers.se

Department of Mathematical Sciences, Chalmers University of Technology and University of Gothenburg, SE 41296 Gothenburg, Sweden 